\title{
Controlling for spatial variability in single site recordings in an in vitro hippocampal preparation with a spontaneous rhythm Jesse Gillis*1,2, Liang Zhang ${ }^{2,3}$ and Frances Skinner ${ }^{1,2,3,4}$
}

\author{
Address: ${ }^{1}$ Department of Physiology, University of Toronto, Toronto, Ontario, Canada, M5S 1A8, ${ }^{2}$ Division of Fundamental Neurobiology, \\ Toronto Western Research Institute, Toronto, Ontario, Canada, M5T 2S8, ${ }^{3}$ Department of Medicine (Neurology), University of Toronto, Toronto, \\ Ontario, Canada, M5S 1A8 and ${ }^{4}$ Institute of Biomaterials and Biomedical Engineering, University of Toronto, Toronto, Ontario, Canada, M5T 2S8 \\ Email: Jesse Gillis* - jaghippocampus@gmail.com \\ * Corresponding author
}

from Sixteenth Annual Computational Neuroscience Meeting: CNS*2007

Toronto, Canada. 7-12 July 2007

Published: 6 July 2007

BMC Neuroscience 2007, 8(Suppl 2):S3 doi:10.1 I86/I47I-2202-8-S2-S3

(c) 2007 Gillis et al; licensee BioMed Central Ltd.

An important consideration in analyzing the time-frequency rhythms of hippocampal local field potentials is to what extent changes in time of a single site signal actually reflect spatial summation of two wholly separate signals. Signals observed could be the result of the spatial summation of different activities. For example, the duration of activities observed could be due to two shorter durations overlapping. More broadly, it is a source of concern if the hippocampal rhythms do not have well defined spatial properties (preferably fairly homogenous) since a single signal has no control for this variable.

To that end, we examine the behaviour of a spontaneous hippocampal rhythm over time as it varies spatially within an intact whole hippocampus preparation. We also examine the response to a high frequency stimulation protocol of $80 \mathrm{~Hz}$. Using four simultaneous rostral to caudal recordings of two to five minutes, we characterize this changing rhythmic activity according to clustered patterns of activity in its time-frequency distribution. Our data set consists of 40 extracellular recordings. The frequency distribution change in response to stimulation was observed. We calculate the spatial variance of the data for each unit of time. This allows us to link the time-frequency data to a level of spatial variance. The variance of the signal in time was defined to be the variance in the mean frequencies of the signal resultant from partitioning each time-frequency epoch into thirds. That is, a sliding 0.5 second temporal resolution was divided into thirds and the vari- ance in the mean frequencies calculated. The change in the signal in time was then compared to the change in signal in space to determine whether there were any significant correlations.

There is a distinct relationship between the variance among simultaneous rostral caudal recordings and the non-stationarity present in each of those spatially distinct recordings. The peak values nearly follow the $y=-x$ line. Functional biological rhythms in the hippocampus are commonly both non-stationary and coherent, making this inverse relationship more intuitive. Consider that the hippocampal recording has two components, one of which is stationary background noise and one of which is the nonstationary signal of interest. Our finding, then, is that the noise is uncorrelated spatially and signal is correlated spatially. In this case, when the signal is low, the non-stationarity would be low and the spatial variance would be high. This is a very useful property for single site recordings because it means that the more physiologically interesting (nonstationary) the recording, the less we need to be concerned that spatial summation is a problem. 